\title{
Consumo de drogas en menores residentes en un área urbana pequeña
}

\section{Drug use in children resident in a small urban area}

\author{
Xavier Pugès Romagosa*; Agustín Vilardebó \\ Clascì ${ }^{*}$; Miquel Angel Martinez Adell*; \\ Patricia García de Olalla ${ }^{* *, * * *}$, Josep Maria Jansà \\ LÓPEZ DEL VALLADO ${ }^{\star \star, * \star *, * \star * *}$.
}

\author{
* Consorci Sanitari del Maresme. \\ **Servicio de Epidemiología. Agència de Salut Pública de \\ Barcelona. \\ *** Investigador Ciber de Epidemiología y Salud Pública \\ (CIBERESP).

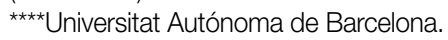

Enviar correspondencia a:

Xavier Pugès Romagosa.

Servicio de Pediatría. Área Básica de Argentona.

C/ Joan Fuster i Ortells 108310 Argentona (Barcelona).

E-mail:11816xpr@comb.es

\section{RESUMEN}

Antecedentes. El comportamiento frente al consumo de sustancias potencialmente adictivas puede estar influido por el lugar de residencia del joven.

Objetivo. Conocer la frecuencia y las caracteristicas del consumo de taba$\mathrm{CO}$, alcohol, cannabis en los alumnos de Educación Secundaria Obligatoria (ESO) de un área urbana pequeña durante el curso 2008-09.

Métodos. Estudio transversal. Se administró un cuestionario autocumplimentado a los alumnos. Se escogieron variables sociodemográficas relacionadas con el consumo.

Resultados. El 88\% de los alumnos respondieron la encuesta (393). La prevalencia de consumo de tabaco fue del $48 \%$, el $16 \%$ declararon fumar diariamente. El $44 \%$ de los alumnos de tercero y el $52 \%$ de los de cuarto consumían alcohol los fines de semana, un 35\% del total se habían emborrachado alguna vez. El 30\% habia consumido cannabis, alcanzando más del 50\% en tercero. De éstos, el 23\% lo hizo en el último mes. La media de edad de inicio en el consumo de cannabis se produjo a los 13 años. El 80\% de los encuestados refirieron no haber pagado para su consumo la primera vez.

Conclusiones. El consumo de alcohol, tabaco y cannabis se inicia en nuestro medio entre los 12 y los 14 años, y con frecuencias ligeramente superiores a las encontradas en grandes urbes. A pesar de la ilegalidad de la venta de tabaco y alcohol a menores, los adolescentes siguen adquiriéndolos. Es fundamental que los profesionales de atención primaria, educadores y la familia tomen conciencia de la importancia que tienen en la detección precoz del consumo de estas sustancias, ya que la prevención de las drogodependencias es tanto más eficaz cuanto antes se detecta el riesgo.

Palabras clave: adolescente, alcohol, tabaco, cannabis, área urbana pequeña.

\section{ABSTRACT}

Background. The use of potentially addictive substances may be influenced by the young person's place of residence.

Objective. To determine the frequency and characteristics of tobacco, alcohol and cannabis use in secondary school pupils in a small urban area during the academic year 2008-09.

Methods. Cross-sectional survey. Socio-demographic data related to tobacco, alcohol and cannabis use were gathered by means of a confidential self-administered questionnaire.

Results. The survey was responded to by $88 \%$ of the pupils (393). Prevalence of tobacco use was $48 \%, 16 \%$ of whom smoked every day. Forty four percent of third grade pupils and fifty two per cent of forth-graders, did consume alcohol on the week ends. Thirty-five percent said that they had been drunk on at least one occasion, while $30 \%$ of the pupils had tried cannabis, and $50 \%$ of third-graders. Among those who had used cannabis, 23\% had done so in the previous month. At onset of cannabis use was 13 years. Almost $80 \%$ of those surveyed said they had not paid for the first drugs they used. Conclusions. The use of legal and illegal drugs for those in our sample begins between the ages of 12 and 14 years, representing slightly higher frequencies than those found in big cities. Despite the illegality of the sale of tobacco and alcohol to minors, Spanish teenagers continue to obtain them. It is essential for primary care professionals, educators and families to be aware of the importance of early detection of the use of psychoactive substances. The prevention of drug addiction is more effective the sooner the potential risk is detected.

Key Words: teenager, alcohol, tobacco, cannabis, small urban area 


\section{INTRODUCCIÓN}

L a adolescencia es una etapa de cambio vital que presenta una gran vulnerabilidad condicionada, en parte, por un espíritu experimentador y por las particulares normas sociales del grupo. Una de las características que definen este periodo es la necesidad de experimentación en todos los ámbitos. Muchos comportamientos que son aspectos normales del desarrollo, como la limitada habilidad para evaluar los riesgos, tomar decisiones y mantener las emociones y deseos bajo control, pueden aumentar la tendencia de los adolescentes a experimentar con las drogas ${ }^{1}$. Algunos adolescentes, pueden ceder a las presiones de los amigos que abusan de las drogas, para compartir estas experiencias con ellos. Otros, pueden pensar que si toman ciertas sustancias podrán disminuir su ansiedad ante eventos sociales ${ }^{2}$.

Aunque el consumo de drogas puede producirse a cualquier edad, numerosos estudios muestran que cuanto más temprano se comienza a consumir, mayor es la probabilidad de progresar al abuso. Esto puede ser debido al efecto directo de las drogas sobre el cerebro en su fase de desarrollo; pero también puede ser la expresión de una variedad de factores, tanto biológicos como sociales, que incluyen la susceptibilidad genética, la enfermedad mental, las relaciones familiares inestables, los compañeros y la exposición al abuso físico o sexual ${ }^{3}$.

En numerosas ocasiones el fracaso escolar y la distorsión de la vida familiar y social constituyen signos precoces del consumo de drogas en estas edades. Diversos estudios evidencian que estamos ante consumidores cada vez más jóvenes, para quienes estos consumos son actos triviales, cuyos riesgos minusvaloran ${ }^{4}$.

Las encuestas dirigidas a grupos de población determinados constituyen una herramienta que permiten conocer la dinámica del consumo de drogas en una comunidad. El conocimiento de la edad de inicio, las características de consumo, las percepciones y actitudes delante del consumo de drogas en la población adolescente contribuye a mejorar el conocimiento del problema, así como a diseñar estrategias de intervención que pueden mejorar la prevención ${ }^{5}$, además de ser un elemento indispensable para evaluar y reorientar los programas de prevención.

La mayoria de los estudios publicados suelen realizarse en núcleos urbanos relativamente grandes, mientras que los llevados a cabo en zonas rurales o en poblaciones pequeñas son escasos. Ante la percepción del personal sanitario de un área básica de salud del incremento del consumo problemático de drogas en los adolescentes se acordó, junto con la dirección del centro educativo, realizar una encuesta a los estudiantes de los cuatro cursos de Enseñanza Secundaria Obligatoria (ESO) del único instituto de la población, para recabar información sobre la extensión del consumo y las características de los consumidores de tabaco, alcohol y cannabis. Desde nuestro punto de vista, merecía la pena estudiar el comportamiento frente al consumo en una población pequeña para verificar si se siguen las mismas premisas que en núcleos urbanos más grandes, ya que el comportamiento frente al consumo puede estar influido por el lugar de residencia del adolescente ${ }^{6,7}$ y si ese fuera el caso, la información recogida podría ayudar a adaptar los programas de prevención a las peculiaridades que pudieran existir en este tipo de poblaciones.

\section{MÉTODOS}

Durante el mes de febrero del 2008 se administró un cuestionario a los alumnos de los cuatro cursos de ESO del único instituto de una localidad urbana, situada al norte de Barcelona que cuenta con una población de unos 12.000 habitantes, de los que el 18\% eran menores de 16 años. Más del $90 \%$ de los jóvenes entre los 12 y los 15 años acudian al instituto de la localidad.

Se utilizó el cuestionario de los estudios FRESC de Barcelona, validado y adaptado para el estudio del consumo de drogas más habituales en adolescentes: tabaco, alcohol y cannabis ${ }^{8}$, para algunas de las preguntas relacionadas con el cannabis, se utilizó el cuestionario de estilos de vida del proyecto xkpts.com que incluye preguntas específicas sobre el consumo de cannabis y sus determinantes ${ }^{9}$.

El cuestionario era anónimo y fue repartido por los tutores de cada curso y cumplimentado por los alumnos en horario lectivo.

De los 448 alumnos matriculados, el 87,7\% (393) cumplimentaron la encuesta. El cuestionario recogía variables sociodemográficas: curso, edad, sexo y situación familiar relacionada con la convivencia con uno o ambos progenitores, así como variables relacionadas con el consumo de tabaco, alcohol y cannabis. El cuestionario permitió clasificar a los alumnos en aquellos que no habian probado el tabaco, los que lo habian hecho de manera ocasional (menos de un cigarrillo a la semana) y quienes fumaban diariamente. Las variables relacionadas con el consumo de alcohol permitieron distribuir a los alumnos en aquellos que lo habian probado y los que no. Entre los que habian tomado bebidas alcohólicas, el consumo de alcohol fue cuantificado según los antecedentes de borracheras. En relación con el consumo de cannabis se estudiaron las siguientes variables: la edad del primer consumo, dónde y con quién estaban la primera vez que lo probaron. Se identificó a los alumnos en aquellos que lo habian consumido alguna vez. La frecuencia de consumo fue categorizada con tres valores: a) cuando lo habian probado alguna vez b) en los últimos 12 meses, pero no en el último mes, y c) cuando el consumo había tenido lugar durante el último mes.

Otras variables recogidas en el cuestionario permitieron explorar la accesibilidad y las circunstancias del consumo de tabaco, alcohol y cannabis, así como la percepción de los efectos negativos que estas sustancias pueden tener sobre la salud.

Se realizó un análisis descriptivo de las variables recogidas mediante porcentajes, medias y su desviación estándar (DE). Como prueba de significación estadística se utilizó el Chi-cuadrado de Pearson en la comparación de porcentajes. El análisis estadístico se realizó mediante el paquete estadístico SPSS versión 13. 


\section{RESULTADOS}

Los 393 participantes en el estudio tenían entre 12 y 16 años (media de 14 años); la edad media de los de primer curso fue de 12,3 años (DE =0,49), de 13,3 años (DE:0,64) en segundo, de 14,5 años $(D E=0,74)$ en tercero y de 15,4 años $(D E=0,68)$ en cuarto. El $52,7 \%$ eran chicos, no apreciándose diferencias significativas en la distribución por sexos en ninguno de los cuatro cursos. El 80,9\% (318) de los encuestados convivía con ambos progenitores, mientras que el $13,7 \%$ lo hacía sólo con la madre, un 3,1\% sólo con el padre y el 2,3\% restante con otros familiares.

En la tabla 1 se muestran las variables relacionadas con el consumo de tabaco según el curso. De forma global el porcentaje de chicas $(51,6 \%)$ de ESO que habian probado el tabaco era superior al de los chicos $(45,1 \%)$ aunque la diferencia no fue significativa $(p=0,19)$. Cabe destacar que el $4,7 \%$ de los alumnos de primero admitieron consumir tabaco diariamente y esta frecuencia aumentó hasta el 23,6\% en los alumnos de cuarto (Tabla 1).

Un 67,2\% (48) de los alumnos que tenían una familia monoparental habian consumido tabaco alguna vez, mientras que esta proporción fue del 44\% (139) en aquellos que convivian con ambos padres $(p=0,001)$.

Con relación a la accesibilidad, un $36,4 \%$ de los alumnos de $4^{\circ}$ de ESO manifestaron haber comprado tabaco para su consumo. El 29\% de los alumnos de primero consideraban como muy peligrosa la repercusión del tabaco sobre la salud, mientras que a partir de segundo este porcentaje decrecía hasta el 10\% observado en los de cuarto, sin apreciarse diferencias entre chicos y chicas.

Con relación al consumo de alcohol, el 66,9\% (263) de los alumnos manifestaron haberlo probado alguna vez. Esta frecuencia se incrementaba con la edad. En primero (12 años) el 43,5\% de los alumnos manifestaron haberlo probado, mientras que entre los de cuarto (15 años) este porcentaje alcanzó el 80\% (Tabla 2).

Aunque no se aprecian diferencias de consumo en la distribución según el sexo (el 71,9\% de los chicos y el 70,3\% de las chicas en la ESO habían probado el alcohol), si que se observaron diferencias en relación al lugar de consumo. Así, entre las chicas fue más frecuente el consumo durante el fin de semana $(68,6 \%)$ frente al $53,2 \%$ de los chicos $(p=0,01)$. En cambio en las fiestas familiares eran los chicos $(46,8 \%)$ los que lo consumian con más frecuencia al compararlos con las chicas (38\%), sin que esta diferencia llegue a ser significativa $(p=0,15)$. De manera global, no se apreciaron diferencias significativas $(p=0,11)$ entre los consumidores de fin de semana al compararlos según el tipo de familias. El 65,3\% de los alumnos que consumian alcohol durante el fin de semana tenía una familia monoparental frente el $58,5 \%$ de los que convivían con ambos padres.

El 34,9\% del total de alumnos encuestados declaró haberse emborrachado en más de una ocasión (Tabla 2). Esta proporción ascendió al 50,4\% entre los escolares que habian probado el alcohol. Entre estos, la proporción de chicas $(54,5 \%)$ que refirieron haberse emborrachado en más de una ocasión fue más alta que la de chicos $(46,8 \%)$, sin ser la diferencia significativa $(p=0,21)$. Según el tipo de familia, se observó que el $45,9 \%$ de los alumnos que convivían en una familia monoparental se habian emborrachado en más de una ocasión, mientras que este porcentaje era del 32,9\% si convivian con ambos padres $(p=0,03)$.

En cuanto a la accesibilidad, el 51,8\% de los alumnos de cuarto manifestó haber comprado alcohol para consumo propio. El $77,6 \%$ de los alumnos consideró el consumo de alcohol como moderadamente peligroso, mientras que un $10 \%$ lo consideraba nada peligroso.

Tabla 1. Descripción de las variables relacionadas con el consumo de tabaco según curso académico

\begin{tabular}{|l|c|c|c|c|c|}
\hline & $\begin{array}{c}\text { Primero } \\
85(\%)\end{array}$ & $\begin{array}{c}\text { Segundo } \\
87(\%)\end{array}$ & $\begin{array}{c}\text { Tercero } \\
111(\%)\end{array}$ & $\begin{array}{c}\text { Cuarto } \\
110(\%)\end{array}$ & $\begin{array}{c}\text { Total (\%) } \\
393\end{array}$ \\
\hline Han probado tabaco & & & & & \\
Si & $8(9,4)$ & $37(42,5)$ & $68(61,3)$ & $74(67,3)$ & $187(47,6)$ \\
No & $77(90,6)$ & $47(54)$ & $43(38,7)$ & $35(31,8)$ & $202(51,4)$ \\
NC & & $3(3,5)$ & & $1(0,9)$ & $4(1)$ \\
\hline Fuman diariamente & $4(4,7)$ & $10(11,5)$ & $21(18,9)$ & $26(23,6)$ & $61(15,5)$ \\
\hline Han comprado tabaco & & & & & \\
para consumo propio & & & & & \\
Si & $5(5,9)$ & $11(12,6)$ & $34(30,6)$ & $40(36,4)$ & $90(23)$ \\
No & $80(94,1)$ & $71(81,6)$ & $76(68,5)$ & $64(58,1)$ & $291(74)$ \\
NC & & $5(5,8)$ & $1(0,9)$ & $6(5,5)$ & $12(3)$ \\
\hline Sensación de peligrosidad & & & & & \\
para la salud. & & & & $11(10)$ & $63(16)$ \\
Muy peligroso & $25(29,4)$ & $12(13,8)$ & $15(13,5)$ & $85(77,3)$ & $279(71)$ \\
Moderadamente peligroso & $49(57,6)$ & $66(75,9)$ & $79(71,2)$ & $13(11,8)$ & $48(12,2)$ \\
Nada peligroso & $10(11,8)$ & $8(9,2)$ & $17(15,3)$ & $1(0,9)$ & $3(0,8)$ \\
\hline NC & $1(1,2)$ & $1(1,1)$ & & & \\
\hline
\end{tabular}

$\mathrm{NC}=:$ no contestan

Tabla 2. Variables relacionadas con el consumo de alcohol según curso académico.

\begin{tabular}{|l|c|c|c|c|c|}
\hline & $\begin{array}{c}\text { Primero } \\
85(\%)\end{array}$ & $\begin{array}{c}\text { Segundo } \\
87(\%)\end{array}$ & $\begin{array}{c}\text { Tercero } \\
111(\%)\end{array}$ & $\begin{array}{c}\text { Cuarto } \\
110(\%)\end{array}$ & $\begin{array}{c}\text { Total }(\%) \\
393\end{array}$ \\
\hline Han probado alcohol & & & & & \\
Si & $37(43,5)$ & $53(60,9)$ & $85(76,6)$ & $88(80)$ & $263(66,9)$ \\
No & $47(55,3)$ & $31(35,6)$ & $19(19)$ & $9(8,2)$ & $106(27,1)$ \\
NC & $1(1,2)$ & $3(3,4)$ & $7(6,3)$ & $13(11,8)$ & $24(6,1)$ \\
\hline Donde acostumbran a & & & & & \\
beber & & & & & \\
Fiestas familiares & $21(24,7)$ & $25(28,7)$ & $20(18)$ & $14(12,7)$ & $80(20,3)$ \\
Fin de semana & $6(7)$ & $14(16)$ & $49(44,1)$ & $57(51,8)$ & $126(32,1)$ \\
Fiestas familiaresy fin & $3(3,5)$ & $6(7)$ & $13(11,8)$ & $22(20)$ & $44(11,2)$ \\
semana & & & & & \\
No consumen/NC & $55(64,8)$ & $42(48,3)$ & $29(26,1)$ & $17(15,5)$ & $143(36,4)$ \\
\hline Se han emborrachado & & & & & \\
En diversas ocasiones & $5(5,9)$ & $15(17,3)$ & $57(51,4)$ & $60(54,6)$ & $137(34,9)$ \\
Nunca & $78(91,8)$ & $70(80,5)$ & $54(48,6)$ & $48(43,6)$ & $250(63,6)$ \\
NC & $2(2,3)$ & $2(2,3)$ & & $2(1,8)$ & $6(1,5)$ \\
\hline Han comprado alcohol & & & & & \\
para consumo propio & & & & & \\
Si & $6(7,1)$ & $9(10,3)$ & $42(37,8)$ & $57(51,8)$ & $114(29)$ \\
No & $72(84,7)$ & $75(86,3)$ & $68(61,3)$ & $43(39,1)$ & $258(65,7)$ \\
NC & $7(8,2)$ & $3(3,4)$ & $1(0,9)$ & $10(9,1)$ & $21(5,3)$ \\
\hline Sensación de peligrosidad & & & & & \\
Muy peligroso & $10(11,8)$ & $13(14,9)$ & $9(8,1)$ & $11(10)$ & $43(10,9)$ \\
Moderadamente peligroso & $57(67)$ & $71(81,7)$ & $88(79,3)$ & $89(80,9)$ & $305(77,6)$ \\
Nada peligroso & $14(16,5)$ & $2(2,3)$ & $14(12,6)$ & $8(7,3)$ & $38(9,7)$ \\
NC & $4(4,7)$ & $1(1,1)$ & & $2(1,8)$ & $7(1,8)$ \\
\hline
\end{tabular}

$\mathrm{NC}=:$ no contestan 
Tabla 3. Variables relacionadas con el consumo de cannabis

\begin{tabular}{|c|c|c|c|c|c|}
\hline $\begin{array}{l}\text { Alumnos por curso que } \\
\text { han probado el cannabis }\end{array}$ & $\begin{array}{c}\text { Primero } \\
3 / 85 \\
(3,5 \%) \\
\end{array}$ & $\begin{array}{c}\text { Segundo } \\
11 / 87 \\
(12,6 \%)\end{array}$ & $\begin{array}{c}\text { Tercero } \\
56 / 111 \\
(50,5 \%)\end{array}$ & $\begin{array}{c}\text { Cuarto } \\
49 / 110 \\
(44,5 \%)\end{array}$ & $\begin{array}{l}\text { Total \% } \\
119 / 393 \\
(30,3 \%)\end{array}$ \\
\hline \multicolumn{6}{|l|}{ Donde lo probaron } \\
\hline En la calle o en el parque & & $6(54,5)$ & $30(53,6)$ & $28(57,2)$ & $64(53,8)$ \\
\hline En casa de un amigo & $1(33,3)$ & $3(27,3)$ & $10(17,9)$ & $11(22,4)$ & $25(21,0)$ \\
\hline En su casa & & & $2(3,6)$ & $4(8,2)$ & $6(5,0)$ \\
\hline En la escuela & & $1(9,1)$ & $3(5,4)$ & $2(4,1)$ & $6(5,0)$ \\
\hline De excursión/campamento & & & $4(7,1)$ & $2(4,1)$ & $6(5,0)$ \\
\hline Otros & & & $2(3,6)$ & & $2(1,6)$ \\
\hline NC & $2(66,7)$ & $1(9,1)$ & $5(8,9)$ & $2(4,1)$ & $10(8,4)$ \\
\hline \multicolumn{6}{|l|}{$\begin{array}{l}\text { Con quien estaban la } \\
\text { primera vez }\end{array}$} \\
\hline Solos & & $1(9,1)$ & & & $1(0.9)$ \\
\hline Con amigos & $3(100)$ & $10(90,9)$ & $55(98,2)$ & $46(94)$ & $114(95,8)$ \\
\hline Con hermanos & & & $1(1,8)$ & $2(4)$ & $3(2,4)$ \\
\hline Con un desconocido & & & & $1(2)$ & $1(0,9)$ \\
\hline \multicolumn{6}{|l|}{$\begin{array}{l}\text { Pagaron por consumir la } \\
\text { primera vez }\end{array}$} \\
\hline $\mathrm{Si}$ & $1(33,3)$ & $2(18,2)$ & $6(10,7)$ & $8(16,3)$ & $17(14,3)$ \\
\hline No & $1(33,3)$ & $8(72,8)$ & $48(85,7)$ & $38(77,6)$ & $95(79,8)$ \\
\hline NC & $1(33,3)$ & 1 (9) & $2(3,6)$ & $3(6,1)$ & $7(5,9)$ \\
\hline \multicolumn{6}{|l|}{ Frecuencia consumo } \\
\hline Alguna vez & $1(33,3)$ & $4(36,4)$ & $21(37,5)$ & $21(42,9)$ & $47(39,5)$ \\
\hline Últimos 12 meses & & $3(27,3)$ & $16(28,6)$ & $11(22,4)$ & $30(25,2)$ \\
\hline Últimos 30 días & $1(33,3)$ & $3(27,3)$ & $10(17,9)$ & $13(26,5)$ & $27(22,7)$ \\
\hline NC & $1(33,3)$ & 1 (9) & $9(16)$ & $4(8,2)$ & $15(12,6)$ \\
\hline \multicolumn{6}{|l|}{ Sensación peligrosidad } \\
\hline Muy peligroso & $1(33,3)$ & $2(18,2)$ & $6(10,7)$ & $10(20,4)$ & $19(15,9)$ \\
\hline Moderadamente peligroso & $2(66,6)$ & $7(63,6)$ & $43(76,7)$ & $37(75,5)$ & $89(74,8)$ \\
\hline Nada peligroso & & $2(18,2)$ & $7(12,6)$ & $2(4,1)$ & $11(9,3)$ \\
\hline
\end{tabular}

$\mathrm{NC}=$ no contestan

En la tabla 3 se muestran las características relacionadas con el consumo de cannabis. El 30,3\% (119), el 28,5\% de los chicos y el $32,4 \%$ de las chicas $(p=0,39)$, de los alumnos manifestaron haber probado el cannabis en alguna ocasión. Un pequeño porcentaje $(3,5 \%)$ lo habia consumido en primero, pero el mayor incremento del consumo se observó entre los cursos de segundo (13 años) y tercero (14 años), con prevalencias del $12,6 \%$ y del $50,5 \%$, respectivamente.

Entre los 119 jóvenes que habian consumido cannabis, 27 $(22,7 \%)$ lo habian hecho en el último mes. Observándose una mayor frecuencia entre los chicos $(25,4 \%)$ que entre las chicas $(20 \%)(p=0,48)$. La edad media de inicio de consumo se situó en los 13,5 años $(D E=1,14)$, sin observarse diferencias significativas entre consumidores habituales y ocasionales, ni entre chicos y chicas.

Los alumnos que habian consumido cannabis, habian probado el tabaco en un porcentaje mayor que aquellos que no habian fumado cannabis $(92,4 \%$ vs $28,5 \%)(p<0,001)$. También se observó, que entre los 187 alumnos que habian consumido tabaco, aquellos que lo hacian diariamente habian consumido cannabis en mayor proporción (75\%) que los que fumaban tabaco, pero no diariamente $(50,8 \%)$, siendo esta diferencia significativa $(p=0,001)$.

El 41,4\% de los alumnos que habían consumido alcohol, también, habian probado el cannabis, mientras que sólo el $2,8 \%$ de aquellos que no habían probado el alcohol habían consumido cannabis $(p<0,001)$. De igual manera, la proporción de consumidores de cannabis fue mayor en aquellos que se habian emborrachado $(67,2 \%)$ que entre los que no lo habian hecho nunca $(16,3 \%)$, pero que habian probado el alcohol $(p<0,001)$.

La calle 0 el parque fueron el lugar del primer consumo en el $53,8 \%$, seguido de la casa de un amigo (21\%). No se observaron diferencias entre chicos y chicas. El 95,8\% lo probaron en compañía de amigos. Entre las razones más frecuentes para iniciar el consumo se encontraban la curiosidad $(68 \%)$ y la búsqueda de sensaciones nuevas (31\%).

Se apreciaron diferencias significativas de consumo de cannabis entre los alumnos con familias monoparentales (47\%) al compararlos con los que convivian con los que vivian con los dos miembros de la pareja $(26,4 \%)$ $(p=0,0001)$. También, se observó una frecuencia superior en el consumo habitual (15\%) de los chicos y de las chicas con familia monoparental al compararlos con los que convivían con ambos padres $(6 \%)(p=0,001)$.

El 85\% de los 112 escolares que respondieron la pregunta manifestaron no haber pagado nada para obtener el cannabis cuando lo consumieron por primera vez y el $80 \%$ de ellos consideró que era fácil o muy fácil conseguirlo en nuestro medio.

Entre los que habian consumido cannabis, el 90,7\% pensaban que el cannabis era peligroso para la salud (Tabla 3 ). Un 55,5\% de ellos relataron efectos negativos asociados al su consumo como la pérdida de memoria $(22 \%)$ y la dificultad para estudiar (15\%).

\section{DISCUSIÓN}

Diversos estudios han puesto de manifiesto las diferencias entre los patrones de consumo según el lugar de residencia de los adolescentes ${ }^{10,11}$. Aunque las comparaciones son difíciles, los resultados de este estudio muestran que el consumo de tabaco, alcohol, y cannabis es superior al encontrado en zonas urbanas y rurales del sur de España ${ }^{12}$ y no inferiores a las observadas en zonas urbanas. A pesar de la menor edad de la población de este estudio, se observó que el consumo de tabaco fue ligeramente superior al referido en Cataluña en la encuesta estatal 2006-07 ${ }^{13}$. En esta última, el $46,1 \%$ de los de los encuestados con edades entre los 14 y los 18 años manifestaron haber consumido tabaco, y un $15,8 \%$ lo hacía diariamente. En nuestra población el 47,6\% de los escolares habian probado el tabaco. A los 12 años, lo habían probado un $9 \%$ y a los 13 años ya lo había hecho el 42,5\%. A los 14 años el 19\% fumaba diariamente.

Esta edad temprana de inicio del consumo de tabaco tiene repercusión en el hábito del adulto, pues el inicio prematuro se asocia a un mayor hábito adictivo, objetivado tanto en el número de cigarrillos consumidos diariamente ${ }^{14}$, como en la duración del hábito, lo que conlleva un aumento de la morbi-mortalidad ${ }^{16,17}$. A esto hay que añadir que el tabaco se ha descrito como un potente predictor del consumo de alcohol y de otras drogas ${ }^{18,19}$.

Desde una perspectiva preventiva, es interesante resaltar que en este estudio se observó que los alumnos quefuma- 
ban tabaco diariamente consumian cannabis con mayor frecuencia al compararlos con los que fumaban tabaco, pero no diariamente. Por este motivo cabe pensar que los programas dirigidos a la prevención del consumo del tabaco, probablemente, tengan repercusión en el inicio del consumo de otras sustancias como el alcohol y el cannabis.

Cabe resaltar que el $23 \%$ del total de los alumnos y el 93,4\% de los fumadores diarios, todos ellos menores de 16 años, manifestaron haber adquirido tabaco para consumo propio, lo que indica la gran accesibilidad y el escaso control en la venta de tabaco a menores en nuestro medio, a pesar de la normativa vigente que prohibe la venta de tabaco a menores de 18 años ${ }^{20}$.

En relación con el consumo de alcohol, más de dos tercios $(66,9 \%)$ de los alumnos encuestados refirieron haberlo probado en alguna ocasión. La proporción de alumnos que bebian durante los fines de semana fue del 16\% en los alumnos de segundo curso (13 años), mientras en los de tercero (14 años) y cuarto (15 años) alcanzó el 61\%, porcentaje similar al observado en el resto de Cataluña $(60,9 \%)^{13}$.

En la cultura mediterránea los primeros contactos con el alcohol se producen en edades tempranas y generalmente, en el entorno familiar ${ }^{21}$. Sin embargo, en los últimos años del patrón tradicional de consumo "mediterráneo", caracterizado por su vinculación a las comidas y en el contexto familiar, se ha pasado al modelo anglosajón, en el que se bebe mucho en corto periodo de tiempo y en entornos lúdicos extrafamiliares. Este tipo de patrón es el que se observa de manera habitual en nuestro medio durante los fines de semana, donde los jóvenes beben, generalmente, bebidas de alta graduación, dando lugar a verdaderos "atracones de alcohol"22.

En este estudio se observó que un tercio de los estudiantes de secundaria habian consumido alcohol durante el fin de semana, y que el salto en el incremento tenía lugar entre los 13 y 14 años. Edad que se debería tener en cuenta al implementar estrategias de prevención dirigidas a esta población en nuestra localidad.

Más de la mitad de los alumnos de tercero y cuarto manifestaron haberse emborrachado en más de una ocasión, frecuencia superior a la referida en otros estudios. En Barcelona, en el año 2006, se observó que el 34\% de los alumnos de tercero de ESO manifestaron haberse emborrachado alguna vez ${ }^{23}$. También llama la atención que casi la mitad de los estudiantes de secundaria contestaron que bebian sólo el fin de semana, mientras que el 15,3\% bebian sólo en fiestas familiares. Esto hace suponer que un porcentaje elevado de padres y madres desconocen que sus hijos beben durante los fines de semana, en particular en el caso de las chicas que solían beber con más frecuencia fuera del ámbito familiar. Con relación a los efectos del alcohol sobre la salud cabe señalar que casi el $80 \%$ de los alumnos consideraba el consumo de alcohol como moderadamente peligroso, lo que es consistente con la aceptación social que dicho consumo tiene en los países mediterráneos.

Como con el tabaco, y a pesar que la legislación vigente prohíbe la venta de alcohol a menores de 18 años, más de la mitad de los alumnos de cuarto habian comprado alcohol para autoconsumo, lo que pone en evidencia la alta accesibi- lidad que tiene el alcohol para los menores en nuestro medio y la falta del cumplimiento de la normativa existente.

Como ocurría con el tabaco, el consumo de cannabis fue más frecuente entre los escolares que consumian alcohol. Desde el punto de vista de la prevención es interesante identificar factores asociados al inicio en el uso del cannabis con el fin de prevenir su posterior consumo ${ }^{24,25,26}$.

En el ámbito europeo un tercio de los escolares de 15 y 16 años han probado cannabis, situándose España con una prevalencia del $36 \%$ entre los países a la cabeza de dicho consumo ${ }^{27}$. En nuestro pais, la extensión y la frecuencia de consumo de cannabis aumenta desde los 14 a los 18 años, produciéndose el mayor incremento entre los 14 y los 15 años ${ }^{28}$. En el estudio que presentamos el mayor aumento tuvo lugar entre los 13 y los 14 años. Así, el 50\% de los escolares de 13 años ya habian probado el cannabis. Este porcentaje es sensiblemente superior al 37,5\% observado en la encuesta realizada en Barcelona en el 2006, aproximándose más al 45,8\% detectado en Cataluña, a pesar de la mayor edad de los encuestados.

Al igual que en Barcelona ${ }^{23}$, la edad media de inicio de consumo se situó en los 13,5 años, mientras que a nivel estatal esta edad se sitúa en los 14,6 años ${ }^{13}$. En la mayoría de los países en los que se registraron las tasas más elevadas de consumo frecuente, entre el 5 y el $9 \%$ de los escolares declararon que habian comenzado a consumir cannabis a la edad de 13 años e incluso antes ${ }^{29}$. El inicio del consumo de cannabis antes de los 16 años se ha relacionado con un consumo problemático de ésta y de otras sustancias, con una mayor dependencia, y en general con la aparición y la gravedad de los efectos negativos, debido a los cambios neurobiológicos provocados por el cannabis durante el desarrollo del cerebro $^{30,31}$. Si tenemos en cuenta la edad de inicio del consumo de cannabis y la extensión del mismo, hay motivos para preocuparse, ya que el cannabis puede producir o precipitar problemas de salud mental a largo plazo ${ }^{32}$. En concreto el uso del cannabis en la adolescencia aumenta el riesgo de psicosis e esquizofrenia ${ }^{33,34,35}$.

En el ámbito escolar se ha observado un bajo rendimiento, un aumento del absentismo, abandono prematuro de los estudios y mayor conflictividad ${ }^{36}$.

Son necesarios programas de prevención con el fin de evitar o retrasar el inicio del consumo de drogas y los problemas relacionados con ellas son necesarios. Aunque la evidencia desaconseja las charlas como única actividad preventiva ${ }^{37}$, éstas continúan siendo el principal instrumento de la prevención universal en el ámbito escolar y a escala local en la mayoría de los paises europeos. Sin embargo, programas que se articulan en el enfoque de la influencia social integral, que combinan la formación en habilidades para la vida con educación normativa y adquisición de conocimientos sobre sustancias, parecen haber conseguido la reducción del consumo frecuente de alcohol y cannabis ${ }^{38}$.

La accesibilidad a esta sustancia en nuestro estudio es similar a la descrita en ciudades como en Barcelona, ya que la mayor parte de los escolares (80\%) consideraron que era fácil o muy fácil poder adquirirla, porcentaje superior al 
observado en la encuesta estatal (63\%). Teniendo en cuenta que la disponibilidad a las drogas está descrita como un potente factor relacionado con su consumo es fácil suponer que en esta fase de la vida en la que se unen diferentes factores como la curiosidad, la búsqueda de nuevas sensaciones o la presión de grupo, la probabilidad de iniciarse en el consumo es muy elevada.

Los adolescentes que declararon haber probado el cannabis, consumian con más frecuencia tabaco y alcohol que los no lo habian probado. Diversos estudios, tanto en el ámbito internacional como nacional han puesto de manifiesto la asociación entre el consumo de alcohol, tabaco y cannabis en adolescentes. En ellos casi la totalidad de los adolescentes que habian probado la cocaína y la heroína habian consumido previamente tabaco, alcohol y cannabis ${ }^{39,40}$.

Como en otros estudios, en este trabajo también se observó la asociación del consumo de cannabis en adolescentes y la convivencia en familias monoparentales ${ }^{41}$. Conocer el funcionamiento de la familia del adolescente, identificar grupos 0 individuos vulnerables con oportunidades sociales y personales limitadas, podría facilitar la identificación de los adolescentes con alto riesgo de consumo y de desarrollo de problemas de adicción, en los cuales estrategias de prevención selectivas serían las recomendadas.

Entre las limitaciones de este estudio cabe mencionar que la encuesta fue autocontestada, no obstante se ha demostrado que encuestas de este tipo presentan una aceptable validez y fiabilidad en nuestro entorno ${ }^{42,43}$. Tampoco se ha podido realizar un análisis más detallado de las características del consumo de alcohol debido a la limitación de los indicadores utilizados, pero dada la naturaleza descriptiva del estudio, se han utilizado indicadores que mejor podian caracterizar la magnitud de estos consumos. Otra limitación en la obtención de resultados fue la incapacidad para comparar una serie de variables conductuales, ya que el cuestionario estaba diseñado en dos partes; una dirigida a todos los escolares y una segunda parte dirigida sólo a aquellos que habian consumido cannabis, por lo que no fue posible poner de manifiesto algunas diferencias como la asistencia a clase o la frecuentación de locales de ocio entre ambos grupos.

En conclusión, consideramos que en nuestro ámbito se observa una precocidad en el inicio de consumo de sustancias potencialmente adictivas, legales e ilegales entre los estudiantes de secundaria, en proporciones semejantes a las descritas en otras zonas urbanas de nuestra comunidad. Debido a los peores efectos sobre la salud que una edad temprana de consumo conlleva, es necesario, desde nuestro punto de vista, disminuir la tolerancia social que tanto el alcohol, el tabaco y el cannabis tienen en nuestra sociedad y erradicar la idea de inocuidad que tienen los jóvenes, padres, educadores e incluso profesionales de la salud sobre estas drogas y en particular con respecto al cannabis.

A pesar de que la magnitud del problema es similar a la encontrada en grandes urbes, los recursos de prevención en áreas más pequeñas suelen ser escasos y a veces inexistentes, por lo que son necesarias iniciativas que promuevan la puesta en marcha de programas de prevención que comprometan a todos los agentes, tanto del ámbito familiar (padres y tutores) implicándoles de forma activa en los programas de prevención; como del ámbito social e institucional (asistentes sociales, educadores, sanitarios, administradores locales...) con el objetivo de retardar al máximo el inicio de este consumo. Es fundamental que los profesionales de atención primaria (médicos de familia, pediatras, enfermeras) junto con la familia tomen conciencia de la importancia que tienen en la detección precoz del consumo de sustancias psicoactivas en adolescentes, ya que la atención a las drogodependencias es tanto más eficaz cuanto antes se detecta.

\section{AGRADECIMIENTOS}

A la dirección del centro, profesores y alumnos por su colaboración.

\section{REFERENCIAS}

1. Zarco J. Drogas: ¿Cómo actuar? Bol Pediatr 2007;47 (supl.1) 45-47.

2. Frazkowiak P. Risk taking and adolescent development. The functions of smoking and alcohol consumption in adolescence and its consequences for prevention. Health Prom 1987;2:51-61.

3. Ullman SE, Najdowski CJ, Filipas HH. Child sexual abuse, posttraumatic stress disorder, and substance use: predictors of revictimization in adult sexual assault survivors. J Child Sex Abus 2009;18:367-385.

4. Guía sobre drogas. 2007. Ministerio de Sanidad y Consumo, Delegación del Gobierno para el Plan Nacional sobre Drogas. Disponible en: htpp//www.pnsd.msc.es.

5. Martínez Álvarez J Garcia González J, Domingo Gutiérrez M. Consumo de alcohol, tabaco y drogas en adolescentes. Atención Primaria 1996;7:383-385.

6. Pérez Milena $A$, Martínez ML, Pérez R, Picossi S, Del Moral PJ, Milena JA. Consumo de sustancias tóxicas durante la adolescencia. Diferencias entre el medio rural y el urbano. Atención Primaria 2004;34:177.

7. Lambert D, Gale JA, Hartley D. Substance abuse by youth and young adults in rural America. J Rural Health 2008;24:221-228.

8. Nebot M, Jiménez E, Ariza $C$, Tomas Z. Tendencias en el consumo de tabaco alcohol y cannabis en los adolescentes de Barcelona entre 1987 y 2004. Med Clin (Barc) 2006;126:157-159.

9. Sánchez-Martínez F, Ariza $C$, Pérez $A$, Diéguez $M$, López MJ, Nebot M. Evaluación de proceso del programa escolar de prevención del consumo de cánnabis en adolescentes "xkpts. com". Adicciones 2010 (en prensa).

10. Donnermeyer JF, Scheer SD. An analysis of substance use among adolescents from smaller places. J Rural Health 2001;17:105-113.

11. González Lama J, Calvo Fernández JR, Prats León P. Epidemiological study of risk behaviour in adolescents at school in two populations, semi-rural and urban. Aten Primaria. 2002;30:214-19.

12. Pérez Milena A, Ramírez Segura EM, Jiménez Pulido I, Leal Heimling FJ, Martínez Fernández MåL, Pérez Milena R. Diferencias 
en el consumo urbano y rural de alcohol, tabaco y drogas en adolescentes. Med Fam Andal 2008;9:10-17.

13. Ministerio de Sanidad y Consumo, Secretaria General de Sanidad, Delegación del Gobierno para el Plan Nacional sobre Drogas. Informe de la encuesta estatal sobre uso de drogas en estudiantes de enseñanzas secundarias (ESTUDES) 20062007. En: http://www.pnsd.msc.es/Categoria2/observa/pdf/ Estudes2006-2007.pdf.

14. Fernandez E, Schiaffino $A$, La Vecchia $C$, Borràs JM, Nebot $M$. Age at starting smoking and number of cigarettes smoked in Catalonia, Spain. Prev Med 1999;28:361-366.

15. Hill DJ, White VM, Williams RM. Tobacco and alcohol use among Australian secondary school students in 1990. Med J Aust 1993;158:228-234.

16. Auseums MA, Mesters I, Van breukelen G. Do Ducth 11-12 years old who never smoke, smoke experimentally or smoke regularly has different demographic backgrounds and perceptions of smoking. Eur J Public Health 2003;13:160-167.

17. Okoli CT, Richardson CG, Ratner PA, Johnson JL. Non-smoking youths' "perceived" addiction to tobacco is associated with their susceptibility to future smoking. Addict Behav 2009;34:10101016.

18. Torabi MR, Bailey WJ, Majd Jobberi M. Cigarette smoking as a predictor of alcohol and other drug use by children and adolescents: evidence of the "gateway drug effect". J Sch Health 1993;63:302-306.

19. Tarter RE,Vanyukov M, Kirisci L, Reynols M, Clark DB. Predictors of marijuana use in adolescents before and after licit drug use: examination of the gateway hypothesis. Am J Psychiatry 2006;163:2134-2140.

20. 21261 LEY 28/2005, de 26 de diciembre, de medidas sanitarias frente al tabaquismo y reguladora de la venta, el suministro, el consumo y la publicidad de los productos del tabaco. BOE núm. 309 2005;42241-42250.

21. Zarco Montejo J, Caudevilla Galligo F, López Santiago A, Álvarez Mazariegos JA, Martínez Osorio S. El papel de la atención primaria ante los problemas de salud relacionados con el consumo de drogas. 2007. Sociedad Española de Medicina de Familiar y Comunitaria. Disponible en: http://www.pnsd.msc.es/Categoria2/ publica/pdf/AtencionPrimariaDrogas.pdf.

22. Rodríguez Martos A. ¿Por qué es tan difícil legislar sobre el alcohol en España? Adicciones 2007;19:325-531.

23. Morales $E_{1}$ Ariza $C_{1}$ Nebot M. Consumo de cannabis en los estudiantes de secundaria de Barcelona: inicio en el consumo, efectos experimentados y expectativas. Gac Sanit 2008;22:321-329.

24. Martínez Álvarez J, Garcia González J, Domingo Gutiérrez M, Machin Fernández AJ. Consumo de alcohol, tabaco y drogas en adolescentes. Atención primaria 1996;7:383-385.

25. Von Sydow K, Lieb R, Pfister H, Hofler M, Wittchen HU. What predicts incident use of cannabis and progression to abuse and dependence? A 4-year prospective examination of risk factors in a community sample of adolescents and young adults. Drug Alcohol Depend 2002;68:49-64.

26. Campbell IG, Feinberg I. Longitudinal trajectories of non-rapid eye movement delta and theta EEG as indicators of adolescent brain maturation. Proc Natl Acad Sci USA 2009;106:5177-5180.

27. Informe ESPAD 2007.Consumo de sustancias entre escolares de 35 paises europeos. Disponible en: http://www.emcdda.europa. eu/attachements.cfm/att_77168_ES_ESPAD_summary_ES.pdf.
28. Ministerio de Sanidad y Politica Social, Delegación del Gobierno para el Plan Nacional sobre Drogas. Informe de la encuesta estatal sobre uso de drogas en estudiantes de enseñanzas secundarias (ESTUDES) 2008. Disponible en: http://www.pnsd. msc.es/Categoria2/observa/pdf/Estudes2008_Web.pdf.

29. Observatorio Europeo de las Drogas y las Toxicomanías (OEDT), 2009. Informe Anual 2009: El estado del problema de las drogas en Europa. Oficina para las Publicaciones Oficiales de las Comunidades Europeas. Disponible en: http://www.emcdda. europa.eu/attachements.cfm/att_93236_ES_EMCDDA_AR2009_ ES.pdf

30. Ehrenreich H, Rinn T, Kunert HJ, Moeller MR, Poser W, Schilling $L$, et al. Specific attentional dysfunction in adults following early start of cannabis use. Psychopharmacology 1999;142:295-301.

31. Pistis M, Serra S, Pillolla G, Melis M, Muntoni AL, Gessa GL. Adolescent exposure to cannabinoids induces long-lasting changes in the response to drugs of abuse of rat midbrain dopamine neurons. Biol Psychiatry 2004;56:86-94.

32. De Irala J, Ruiz-Canela M, Martínez-González MA. Causal relationship between cannabis use and psychotic symptoms or depression. Should we wait and see? A public health perspective. Med Sci Monit 2005;11:RA355-58.

33. Henquet C, Krabbendam L, Spauwen J, Kalan C, Lieb R, Wittchen HU, et al. Prospective cohort study of cannabis use, predisposition for psychosis, and psychotic symptoms in young people. BMJ 2005;330-311.

34. González-Pinto A, Vega $P$, Ibáñez B, Mosquera F, Barbeito $S$, Gutiérrez M, Ruiz de Azúa S, Ruiz I, Vieta E. Impact of cannabis and other drugs on age at onset of psychosis. J Clin Psychiatry 2008;69:1210-1216.

35. Arias Horcajadas F. A review about cannabis use like as risk factor of schizophrenia. Adicciones 2007;19:191-203.

36. Fergusson DM, Horwood LJ, Beautrais AL. Cannabis and educational achievement. Addiction 2003;98:1681-1692.

37. Bruvold WH. A meta-analysis of adolescent smoking prevention programs. Am J Public Health 1993; 83:872-880.

38. Prevención del Abuso de Sustancias entre el alumnado. European Drug addiction prevention trial. Disponible en:http://www.edex. es/Temporal/eudap/castellano.pdf.

39. Amos A, Wiltshire $S$, Bostock $Y$, Haw $S$, McNeill A. "You can't go without a fag you need it for your hash". A qualitative exploration of smoking, cannabis and young people. Addiction 2004;99:77-81.

40. Wagner FA, Anthony JC. Into the world of illegal drug use: exposure opportunity and other mechanisms linking the use of alcohol, tobacco, marijuana, and cocaine. Am J Epidemiol 2002; 155:918-925.

41. Guxens $M$, Nebot $M$, Ariza $C$. Age and sex differences in factors associated with the onset of cannabis use: a cohort study. Drug Alcohol Depend 2007;88:234-243.

42. Comin E, Torrubia R, Mor J, Villalbi JR, Nebot M. Fiabililidad de un cuestionario auto administrado para investigar el nivel de ejercicio y consumo de tabaco y de alcohol entre escolares. Med Clin (Bna) 1997;108:293-298.

43. Moncada A, Pérez K. Fiabilidad y validez del autoinforme sobre el consumo de drogas en la población escolarizada de secundaria. Gac Sanit 2001;15:406-413 
